\title{
CONVOLUTION OF GENERALIZED VECTOR MEASURES
}

\author{
OLLI MARTIKAINEN
}

Weakly compact operators from $C^{*}$-algebras into Banach spaces are called generalized vector measures. We construct their convolution in such a way that it generalizes the convolution of regular Borel vector measures as well as that of noncommutative analogues of vector measures.

\section{Introduction}

The concept of the convolution of Banach space valued regular Borel vector measures was introducted by M. Duchoň in [4]. Let us apply his definition to the case of a locally compact (Hausdorff) semigroup $X$ with a separately continuous composition (compare [19, p. 131]). If $\bar{\mu}: \mathscr{B}(X) \rightarrow E_{1}$ and $\bar{v}: \mathscr{B}(X) \rightarrow E_{2}$ are Banach space valued regular Borel vector measures, their convolution is the regular Borel vector measure $\bar{\mu} * \bar{v}: \mathscr{B}(X) \rightarrow E_{1} \hat{\hat{\otimes}} E_{2}$ defined by

$$
\int_{X} f d \bar{\mu} * \bar{v}=\int_{X \times X} f(s t) d(\bar{\mu} \otimes \bar{v})(s, t) \quad\left(f \in C_{0}(X)\right),
$$

where we use the generalized Riesz representation theorem (see [5, Theorem VI.7.3]). In other words, if $\Phi: C_{0}(X) \rightarrow E_{1}$ and $\Psi: C_{0}(X) \rightarrow E_{2}$ are the weakly compact operators corresponding to $\bar{\mu}$ and $\bar{v}$, respectively, the weakly compact operator $\Phi * \Psi: C_{0}(X) \rightarrow E_{1} \hat{\hat{\otimes}} E_{2}$ corresponding to $\bar{\mu} * \bar{\nu}$ is the combined mapping $(\underset{\varepsilon, \varepsilon}{\otimes} \Psi)^{\prime \prime} \circ P$, where $P: C_{0}(X) \rightarrow C_{0}(X \times X)^{\prime \prime}$ is defined by $(P(f))(s, t)=f(s t)$ $\left(f \in C_{0}(X) ; s, t \in X\right)$. In fact, $P$ is the restriction to $C_{0}(X)$ of the transpose of the mapping $\tilde{M}: M(X \times X) \rightarrow M(X)$ given by

$$
\int_{X} f d \tilde{M}(\mu)=\int_{X \times X} f(s t) d \mu(s, t) \quad\left(f \in C_{0}(X), \quad \mu \in M(X \times X)\right) .
$$

Let $G$ be a locally compact group and $C^{*}(G)$ its group $C^{*}$-algebra. If $G$ is commutative, $C^{*}(G)$ can be identified with $C_{0}(\hat{G})$, where $\hat{G}$ is the dual group of $G$. Then regular Borel vector measures on $\hat{G}$ correspond to Banach space valued weakly compact operators on $C^{*}(G)$. In the general, not necessarily commutative case, weakly 
compact operators from $C^{*}(G)$ into a Banach space are called noncommutative analogues of vector measures [20]. If $\Phi$ and $\Psi$ are noncommutative analogues of vector measures on $C^{*}(G)$, their convolution can be defined naturally to be the composed mapping $(\underset{v, \varepsilon}{\otimes} \Psi)^{\prime \prime} \circ p_{I}$, where $v$ is the $C^{*}$-norm on $C^{*}(G) \otimes C^{*}(G)$ induced by $C^{*}(G \times G)$ and $p_{I}: C^{*}(G) \rightarrow C^{*}(G \times G)^{\prime \prime}$ is the restriction to $C^{*}(G)$ of the transpose $\tilde{m}^{\prime}$ of the operator $\tilde{m}: C^{*}(G \times G)^{\prime} \rightarrow C^{*}(G)^{\prime}$ given by

$$
(\tilde{m}(u))(s)=u(s, s) \quad\left(s \in G, u \in B(G \times G)=C^{*}(G \times G)^{\prime}\right)
$$

(see [14]). In this case we must, however, assume that $\underset{v, \varepsilon}{\otimes} \Psi$ is a weakly compact operator. This concept of convolution is consistent with the notion of a Fourier transform introduced by $\mathrm{K}$. Ylinen in [20].

To unify and generalize the two examples above we shall study an arbitrary $C^{*}$-algebra instead of the $C^{*}$-algebras $C_{0}(X)$ and $C^{*}(G)$. We observe that the aforementioned maps $\tilde{M}$ and $\tilde{m}$ are in fact extensions of the multiplications of $M(X)$ and $B(G)$ :

$$
\begin{gathered}
\tilde{M}(\mu * v)=\mu * v \text { for all } \mu, v \in M(X), \quad \text { and } \\
\tilde{m}(u \otimes v)=u v \text { for all } u, v \in B(G)=C^{*}(G)^{\prime} .
\end{gathered}
$$

A $C^{*}$-algebra $A$ is said to be of type $M^{\prime}$ if there exists a bounded bilinear multiplication on $A^{\prime}$ which can be extended to a bounded linear mapping $m: A^{\prime} \bar{\otimes}_{\beta} A^{\prime} \rightarrow A^{\prime}$, where

$$
m(x \otimes y)=x y \quad\left(x, y \in A^{\prime}\right)
$$

and $\beta$ is the unique crossnorm on $A^{\prime} \otimes A^{\prime}$ dual to every $C^{*}$-norm on $A \otimes A$. We give examples on $C^{*}$-algebras of type $M^{\prime}$ in Section 3.

Weakly compact operators from a $C^{*}$-algebra $A$ into a Banach space will be called generalized vector measures. To define a bilinear convolution for generalized vector measures on $A$ the multiplicative structure of $A^{\prime}$ is clearly a necessary requirement, because the elements of the dual are simply complex valued weakly compact operators on $A$ and so their convolution induces a multiplication on the dual $A^{\prime}$.

If $A$ is of type $M^{\prime}$, and $\Phi$ and $\Psi$ are generalized vector measures on $A$, the convolution $\Phi * \Psi$ can be constructed in a way which extends both the approaches presented above. We shall consider $\Phi * \Psi$ in detail in Section 5. There $\Phi * \Psi$ is defined to be the composition of the product $\Phi \times \Psi$ and $m^{\prime} \mid A$, the transpose of $m$ restricted to $A$. The product $\Phi \times \Psi$ of $\Phi$ and $\Psi$ is assumed to be weakly compact and it extends $\Phi_{\mu, \varepsilon} \Psi$ and $\Phi^{\prime \prime} \underset{\mu, \varepsilon}{\otimes} \Psi^{\prime \prime}$ to the $W^{*}$-tensor product $A^{\prime \prime} \bar{\otimes} A^{\prime \prime}$. This extension is necessary since the range of $m^{\prime} \mid A$ is contained in $\left(A^{\prime} \bar{\otimes}_{\beta} A^{\prime}\right)^{\prime}=A^{\prime \prime} \bar{\otimes} A^{\prime \prime}$ but not always in $A \otimes_{\mu} A[14$, p. 31]. We shall study $\Phi \times \Psi$ in Section 4.

Finally, in the end of Section 5, we shall examine the Banach algebra valued convolution and conclude with the following application. Let $A$ be a $C^{*}$-algebra of 
type $M^{\prime}$ such that $A^{\prime}$ is commutative. With some restrictive assumptions the space of compact operators from $A$ into a commutative Banach algebra $B$ can be equipped with convolution which makes it a commutative Banach algebra, and its spectrum becomes equal to the cartesian product of the spectra of $A^{\prime}$ and $B$.

\section{Preliminaries}

We shall use the results presented in this section throughout the text, usually without explicit reference. The scalar field will always be the complex field $C$. We shall denote Banach spaces by $E, E_{1}$ and $E_{2}$ and $C^{*}$-algebras by $A, A_{1}$ and $A_{2}$. The (topological) dual of $E$ is denoted by $E^{\prime}$ and the closed unit ball of $E$ by $E_{0}$. The topology $\sigma\left(E, E^{\prime}\right)$ is called the weak (or $w$-) topology of $E$ and $\sigma\left(E^{\prime}, E\right)$ the $w^{*}$ topology of $E^{\prime}$. We say that a mapping $T$ from $E_{1}$ into $E_{2}$ is an operator if it is linear and continuous. Its transpose from $E_{2}^{\prime}$ into $E_{1}^{\prime}$ is denoted by $T^{\prime}$. If $T$ maps the closed unit ball of $E_{1}$ into a relatively weakly compact subset of $E_{2}$, we call $T$ weakly compact. In particular, we shall call weakly compact operators from a $C^{*}$-algebra into a Banach space generalized vector measures. The canonical embedding from $E$ into its bidual $E^{\prime \prime}$ will be denoted by $J_{E}$. The symbol $\circ$ stands for the composition of mappings and the symbol $*$ is used for the convolution. The basic theory of Banach spaces is assumed to be known. As for Banach algebras, Banach * -algebras, $C^{*}$-algebras and $W^{*}$ - (i.e. von Neumann) algebras, we refer to [3], [15] and [18]. Especially, we shall always identify the bidual $A^{\prime \prime}$ of a $C^{*}$-algebra $A$ with the so-called enveloping von Neumann algebra of $A$ [3, p. 265]. The multiplication on $A^{\prime \prime}$ is then the Arens product on $A^{\prime \prime}[2, \mathrm{p} .869]$ and the involution is the dual involution with respect to $A^{\prime}\left[3\right.$, p. 6]. The canonical embedding $J_{A}$ of $A$ into $A^{\prime \prime}$ is a $*$-homomorphism. If $x \in A$ and $f \in A^{\prime}$, we usually write $\langle f, x\rangle$ or $\langle x, f\rangle$ instead of $f(x)$. Kaplansky's density theorem is used in the following form: if $A_{1}$ is a $w^{*}$-dense $*$-subalgebra of a von Neumann algebra $A$, then $\left(A_{1}\right)_{0}$ is $w^{*}$-dense in $A_{0}$ (see [18, p. 82]).

Let $E_{1}$ and $E_{2}$ be Banach spaces. The tensor product $E_{1} \otimes E_{2}$ equipped with a norm $\alpha$ is denoted by $E_{1} \otimes_{\alpha} E_{2}$ and its completion by $E_{1} \bar{\otimes}_{\alpha} E_{2}$. For the $\varepsilon$ - and $\pi$-norms the completion is, however, denoted by $E_{1} \hat{\otimes} E_{2}$ and $E_{1} \hat{\otimes} E_{2}$, respectively. For the theory of tensor products of $C^{*}$-algebras we refer to [18]. In particular, we denote the injective and projective $C^{*}$-norms by $\mu$ and $v$, respectively. For each $C^{*}$-norm $\alpha$ we have $\varepsilon \leqq \mu \leqq \alpha \leqq \nu \leqq \pi$; see [9, pp. 11, 16 and 18] and [18, pp. 206-208 and 216]. Let $\Phi$ and $\Psi$ be operators. If $\Phi \otimes \Psi$ is continuous when the tensor product of the initial (or final) spaces is equipped with the norm $\alpha$ (or $\beta$ ), $\Phi \otimes \Psi$ can be extended to the completions, and we denote this extension by $\Phi \otimes \otimes_{\alpha, \beta} \Psi$.

For the $W^{*}$-tensor product $M \bar{\otimes} N$ of $W^{*}$-algebras $M$ and $N$ we refer to [18, p. 221]. The predual $(M \bar{\otimes} N)_{*}$ of $M \bar{\otimes} N$ is $M_{*} \bar{\otimes}_{\beta} N_{*}$, where $\beta$ is the dual norm on $M_{*} \otimes N_{*}$ induced by $\left(M \bar{\otimes}_{\mu} N\right)^{\prime}$. For $C^{*}$-algebras $A_{1}$ and $A_{2}$ the predual of $A_{1}^{\prime \prime} \bar{\otimes} A_{2}^{\prime \prime}$ is $A_{1}^{\prime} \bar{\otimes}_{\beta} A_{2}^{\prime}$. 
Lemma 2.1. The dual norm on $A_{1}^{\prime} \otimes A_{2}^{\prime}$ of any $C^{*}$-norm on $A_{1} \otimes A_{2}$ agrees with $\beta$.

Proof. By $\left[18\right.$, p. 209] the dual norms of all $C^{*}$-norms on $A_{1} \otimes A_{2}$ agree on $A_{1}^{\prime} \otimes A_{2}^{\prime}$. On the other hand, $A_{1} \bar{\otimes}_{\mu} A_{2}$ can be regarded as a $w^{*}$-dense subspace of $A_{1}^{\prime \prime} \bar{\otimes} A_{2}^{\prime \prime}\left[18\right.$, pp. 208 and 211], and so $\left(A_{1} \bar{\otimes}_{\mu} A_{2}\right)_{0}$ is $w^{*}$-dense in $\left(A_{1}^{\prime \prime} \bar{\otimes} A_{2}^{\prime \prime}\right)_{0}$ by Kaplansky's density theorem. Hence $\beta$ is equal to the dual norm $\mu^{\prime}$.

Let $X$ be a locally compact (Hausdorff) topological space. The dual of the $C^{*}$-algebra $C_{0}(X)$ of bounded continuous complex functions (with the supremum norm) on $X$ vanishing at infinity is identified as usual with the Banach space of bounded regular complex Borel measures (with the total variation norm) on $X$, and denoted by $M(X)$. Let $G$ be a locally compact group with a fixed left Haar measure $d s$. For any function $f \in C_{0}(G)$ we write $\tilde{f}(x)=\overline{f\left(x^{-1}\right)}(x \in G)$. If $\mu \in M(X)$, the measure $\mu^{*}$ is defined by $\mu^{*}(f)=\overline{\mu(\tilde{f})}\left(f \in C_{0}(G)\right)$. We denote by $L^{1}(G)$ the Banach space of the (equivalence classes of) complex Borel functions on $G$ integrable with respect to $d s$. We shall embed $L^{1}(G)$ in the usual way into $M(G)$. Equipped with the convolution product and the involution $\mu \mapsto \mu^{*}$ the space $M(G)$ is a Banach *-algebra having $L^{1}(G)$ as a closed *-ideal (see e.g. [3, p. 282] and [7, p. 185]). The enveloping $C^{*}$-algebra of $L^{1}(G)$ is denoted by $C^{*}(G)$ and called the group $C^{*}$-algebra of $G[3$, pp. 48,303]. The enveloping von Neumann algebra of $C^{*}(G)$ is designated by $W^{*}(G)$ and it is *-isomorphic to the bidual $C^{*}(G)^{\prime \prime}$ of $C^{*}(G)$. The set of linear combinations of positive definite functions on $G$ will be denoted by $B(G)$ and called the Fourier-Stieltjes algebra of $G$. By [7, p. 192] $B(G)$ can be identified with the dual of the group $C^{*}$-algebra $C^{*}(G)$ by the mapping $\tau$ which satisfies

$$
\langle\tau(u), f\rangle=\int_{G} u(s) f(s) d s \quad\left(u \in B(G), f \in L^{1}(G)\right) .
$$

We also remark that for the cartesian products $X \times X$ and $G \times G$ we have $C_{0}(X \times X)=$ $C_{0}(X) \hat{\otimes} C_{0}(X)$ and $C^{*}(G \times G)=C^{*}(G) \bar{\otimes}_{\nu} C^{*}(G)$ (see [17, p. 357] and [9, p. 13]).

\section{Spaces of type $M_{\alpha}$ and $M^{\prime}$}

As mentioned in the introduction, we shall define the convolution of generalized vector measures $\Phi$ and $\Psi$ on $A$ as the composition of the product generalized vector measure $\Phi \times \Psi$ and the mapping $m^{\prime} \mid A: A \rightarrow A^{\prime \prime} \bar{\otimes} A^{\prime \prime}$, where $m$ is the unique bounded linear mapping from $A^{\prime} \bar{\otimes}_{\beta} A^{\prime}$ into $A^{\prime}$ extending a given bounded bilinear multiplication on $A^{\prime}$. However, it is not always trivial that such a bounded extension $m$ exists. In this section we shall give examples on $C^{*}$-algebras which have this property. To shorten our presentation we give the following definition.

Definition 3.1. Let $E$ be a Banach space equipped with a bounded bilinear multiplication and let $\alpha$ be a norm on $E \otimes E$. Denote by $m$ the linear mapping from 
$E \otimes E$ into $E$ defined by $m(x \otimes y)=x y(x, y \in E)$. We say that $E$ is of type $M_{\alpha}$ if $m$ is bounded on $E \otimes_{\alpha} E$.

It is clear that if $E$ is a Banach space of type $M_{\alpha}$ and $\beta$ is a norm on $E \otimes E$ not less than $\alpha$, then $E$ is of type $M_{\beta}$. Let us have two concrete examples.

Example 3.2. Each Banach algebra $B$ is of type $M_{\pi}$. The estimate $\|m(z)\| \leqq$ $\pi(z) \quad(z \in B \otimes B)$ follows from the inequality $\|x y\| \leqq\|x\|\|y\| \quad(x, y \in B)$.

Example 3.3. Commutative $C^{*}$-algebras are of type $M_{\varepsilon}$. This is a result of the fact that they can be identified with spaces $C_{0}(X)$, where $X$ is locally compact. Now $C_{0}(X) \hat{\otimes} C_{0}(X)=C_{0}(X \times X)$ and

$$
\|m(z)\|=\left\|\sum_{i=1}^{n} f_{i} g_{i}\right\|_{\infty} \leqq\left\|\sum_{i=1}^{n} f_{i} \otimes g_{i}\right\|_{\infty}=\varepsilon(z)
$$

for all $z=\sum_{i=1}^{n} f_{i} \otimes g_{i} \in C_{0}(X) \otimes C_{0}(X)$.

In what follows we shall be dealing with $C^{*}$-algebras whose duals are of type $M_{\beta}$.

Definition 3.4. Let $A$ be a $C^{*}$-algebra, $A^{\prime}$ its Banach space dual and $\beta$ the unique dual norm on $A^{\prime} \otimes A^{\prime}$ of any $C^{*}$-norm on $A \otimes A$. If $A^{\prime}$ is equipped with a bounded bilinear multiplication which makes it a Banach space of type $M_{\beta}$, we say that $A$ is of type $M^{\prime}$.

On $A^{\prime}$ there may exist several multiplications which make $A^{\prime}$ a Banach space of type $\beta$. For instance, if $G$ is a finite group, then $C_{0}(G)^{\prime}=L^{1}(G)$ is of type $M_{\beta}$ with respect to the pointwise multiplication and the convolution product.

In particular, if the multiplication of $A$ or $A^{\prime}$ is commutative, we can develop sufficient conditions for $A$ to be of type $M^{\prime}$.

Assume first that $A$ is a commutative $C^{*}$-algebra such that there is a bounded bilinear multiplication on $A^{\prime}$. Then $A$ can be identified with a space $C_{0}(X)$ for some locally compact space $X$. So, the dual of $A$ is $M(X)$, and since $M(X)$ has the (Grothendieck) metric approximation property, the dual norm $\beta$ on $M(X) \otimes M(X)$ is equal to the $\pi$-norm. Hence $A^{\prime}$ is of type $M_{\beta}$ and $A$ of type $M^{\prime}$. On the other hand, $A^{\prime \prime}$ is also a commutative $C^{*}$-algebra so that it is of type $M^{\prime}$ when $A^{\prime \prime \prime}$ is equipped with one of the Arens extensions of the multiplication of $A^{\prime}$. Similarly, $A^{\prime \prime \prime \prime}$ is of type $M^{\prime}$, and so on. Let us state this as a theorem.

Theorem 3.5. If $A$ is a commutative $C^{*}$-algebra such that its dual has a bounded bilinear multiplication, then $A$ is of type $M^{\prime}$, and so are all its even duals.

Suppose now that $A$ is a $C^{*}$-algebra whose dual $A^{\prime}$ is a commutative Banach algebra. When we define a multiplication on the algebraic tensor product $A^{\prime} \otimes A^{\prime}$ by the natural formula

$$
u v=\sum_{i, j} s_{i} s_{j}^{\prime} \otimes t_{i} t_{j}^{\prime} \quad\left(u=\sum_{i} s_{i} \otimes t_{i}, v=\sum_{j} s_{j}^{\prime} \otimes t_{j}^{\prime}\right)
$$


$A^{\prime} \otimes A^{\prime}$ becomes a commutative algebra, and $m$ is a homomorphism from $A^{\prime} \otimes A^{\prime}$ into $A^{\prime}$. If we assume that $A^{\prime} \bar{\otimes}_{\beta} A^{\prime}$ is a Banach algebra and $m$ can be extended to it, we get the following theorem.

Theorem 3.6. If $A^{\prime}$ is a semisimple commutative Banach algebra such that $A^{\prime} \bar{\otimes}_{\beta} A^{\prime}$ is a Banach algebra and $m$ can be extended to a homomorphism from $A^{\prime} \bar{\otimes}_{\beta} A^{\prime}$ to $A^{\prime}$, then $A$ is of type $M^{\prime}$.

Proof. Since any homomorphism from a Banach algebra into a semisimple commutative Banach algebra is continuous [15, p. 75], the assertion follows straightly.

Corollary 3.7. Let $A^{\prime}$ be a semisimple commutative Banach algebra. Let $\alpha$ be a $C^{*}$-norm and suppose that $\left(A \bar{\otimes}_{\alpha} A\right)^{\prime}$ is a commutative Banach algebra having $A^{\prime} \otimes A^{\prime}$ as a subalgebra. If there is a homomorphism $\tilde{m}:\left(A \bar{\otimes}_{\alpha} A\right)^{\prime} \rightarrow A^{\prime}$ which extends $m$, then $A$ is of type $M^{\prime}$.

We can establish the boundedness of $m: A^{\prime} \otimes_{\beta} A^{\prime} \rightarrow A^{\prime}$ also by studying its transpose. It is well known and easy to see that if $m$ is bounded, then $m^{\prime}(x)=x \otimes x$ for each element $x$ in the spectrum $\sigma\left(A^{\prime}\right)$ of $A^{\prime}$.

Theorem 3.8. Let $A^{\prime}$ be a semisimple commutative Banach algebra such that $\sigma\left(A^{\prime}\right) \cup\{0\}$ is stable under the involution and the multiplication of $A^{\prime \prime}$. If there exists a *-homomorphism $p$ from the closed linear span of $\sigma\left(A^{\prime}\right) \cup\{0\}$ into $A^{\prime \prime} \bar{\otimes} A^{\prime \prime}$ such that

then $A$ is of type $M^{\prime}$.

$$
p(x)=x \otimes x\left(x \in \sigma\left(A^{\prime}\right)\right)
$$

Proof. Denote the linear span of $\sigma\left(A^{\prime}\right) \cup\{0\}$ by $F$ and its norm closure by $\bar{F}$. Since $A^{\prime}$ is semisimple, $F$ is a $w^{*}$-dense involutive subalgebra of $A^{\prime \prime}$. By Kaplansky's density theorem $F \cap\left(A^{\prime \prime}\right)_{0}$ is $w^{*}$-dense in $\left(A^{\prime \prime}\right)_{0}$. Now, $\bar{F}$ is a $C^{*}$-algebra and $p: \bar{F} \rightarrow A^{\prime \prime} \bar{\otimes} A^{\prime \prime}$ is a $*$-homomorphism. Consequently, $p$ is contractive [3, p. 9]. We have for all $w=\sum_{i} u_{i} \otimes v_{i}$ in $A^{\prime} \otimes_{\beta} A^{\prime}$ and $x=\sum_{j} \beta_{j} k_{j}$ in $F\left(\beta_{j} \in \boldsymbol{C}\right.$, $\left.k_{j} \in \sigma\left(A^{\prime}\right)\right)$

$$
\begin{gathered}
\langle m(w), x\rangle=\sum_{i, j} \beta_{j}\left\langle u_{i} v_{i}, k_{j}\right\rangle=\sum_{i, j} \beta_{j}\left\langle u_{i} \otimes v_{i}, k_{j} \otimes k_{j}\right\rangle \\
=\sum_{i, j} \beta_{j}\left\langle u_{i} \otimes v_{i}, p\left(k_{j}\right)\right\rangle=\langle w, p(x)\rangle .
\end{gathered}
$$

Hence we obtain

$$
\|m(w)\|=\sup \left\{|\langle m(w), x\rangle| \mid x \in F \cap\left(A^{\prime \prime}\right)_{0}\right\}=\sup \left\{|\langle w, p(x)\rangle| \mid x \in F \cap\left(A^{\prime \prime}\right)_{0}\right\} \leqq\|w\| .
$$

So $\|m\| \leqq 1$, and $A^{\prime}$ is of type $M_{\beta}$.

If the mapping $p$ in the previous theorem can be extended to a normal *-homomorphism from $A^{\prime \prime}$ into $A^{\prime \prime} \bar{\otimes} A^{\prime \prime}$, then it is necessarily the transpose of $m$.

We conclude this section with examples. 
Example 3.9. Let $X$ be a locally compact semigroup with a separately continuous multiplication. Equip $C_{0}(X)$ with the pointwise multiplication and the involution $f \mapsto \bar{f}$. The convolution of measures defines a bounded bilinear multiplication on the dual $M(X)$ [8]. So, by Theorem 3.5, $C_{0}(X)$ is a $C^{*}$-algebra of type $M^{\prime}$, and so are $C_{0}(X)^{\prime \prime}$, its bidual etc.

Example 3.10. Let $G$ be a locally compact group and $B(G)$ its FourierStieltjes algebra, i.e., the set of linear combinations of continuous positive definite functions on $G$. Identify $B(G)$ with the dual of the group $C^{*}$-algebra $C^{*}(G)$. Equipped with the pointwise multiplication inherited from $B(G)$ and the dual involution, $C^{*}(G)^{\prime}$ is a unital semisimple commutative Banach *algebra. The mapping $\tilde{m}$ : $B(G \times G) \rightarrow B(G)$ given by

$$
(\tilde{m}(u))(s)=u(s, s) \quad(s \in G, u \in B(G \times G))
$$

is a homomorphism and it extends the mapping $m: B(G) \otimes B(G) \rightarrow B(G)$ which corresponds to the pointwise multiplication of $B(G)$. Hence the conditions of Corollary 3.7 are fulfilled and so $C^{*}(G)$ is of type $M^{\prime}$.

Example 3.11. If $A$ is a so-called Hopf- $C^{*}$-algebra [11, Definition 2.2], there exists a $*$-homomorphism $d: A \rightarrow\left(A \bar{\otimes}_{v} A\right)^{\prime \prime}$. The mapping

$$
(x, y) \mapsto d^{\prime}\left(J_{\left(A \bar{\otimes}_{v} A\right)^{\prime}}(x \otimes y)\right) \quad\left(x, y \in A^{\prime}\right)
$$

defines a bilinear multiplication on $A^{\prime}$ which can be extended to a bounded linear mapping $m$ on $A^{\prime} \bar{\otimes}_{\beta} A^{\prime} \subset\left(A \bar{\otimes}_{v} A\right)^{\prime}$. Consequently, $A$ is of type $M^{\prime}$, and $d$ agrees with $m^{\prime} \mid A$.

Example 3.12. If $A^{\prime \prime}$ is a Hopf-von Neumann algebra [6, Definition 3.1], there exists a $*$-homomorphism $d: A \rightarrow A^{\prime \prime} \otimes A^{\prime \prime}$. The mapping

$$
(x, y) d^{\prime}\left(J_{A^{\prime} \otimes_{\beta} A^{\prime}}(x \otimes y)\right) \quad\left(x, y \in A^{\prime}\right)
$$

gives now a bilinear multiplication on $A^{\prime}$ which makes $A$ a $C^{*}$-algebra of type $M^{\prime}$.

\section{The product of generalized vector measures}

Let $\Phi: A_{1} \rightarrow E_{1}$ and $\Psi: A_{2} \rightarrow E_{2}$ be generalized vector measures. For the construction of the convolution of generalized vector measures the operator $\Phi \underset{\mu, \varepsilon}{\otimes} \Psi$ must be extended to the $w^{*}$-tensor product of $A_{1}^{\prime \prime}$ and $A_{2}^{\prime \prime}$.

Theorem 4.1. Let $\Phi: A_{1} \rightarrow E_{1}$ and $\Psi: A_{2} \rightarrow E_{2}$ be generalized vector measures such that $\Phi_{\mu, \varepsilon}^{\otimes} \Psi$ is weakly compact. Denote the operator $\left(\Phi_{\pi, \beta}^{\prime} \Psi^{\prime}\right)^{\prime}$ by $\Phi \times \Psi$. Then $\Phi \times \Psi$ can be regarded as a weakly compact and $w^{*}$-w-continuous operator from 
$A_{1}^{\prime \prime} \bar{\otimes} A_{2}^{\prime \prime}$ into $E_{1} \hat{\hat{\otimes}} E_{2}$. Moreover, $\Phi \times \Psi$ is the only $w^{*}$-w-continuous operator which extends $\Phi \underset{\mu, \varepsilon}{\otimes} \Psi$ (consider $A_{1} \bar{\otimes}_{\mu} A_{2}$ as a subspace of $\left.A_{1}^{\prime \prime} \bar{\otimes} A_{2}^{\prime \prime}\right)$.

Proof. By assumption the operator $\Phi \underset{\mu, \varepsilon}{\otimes} \Psi$ is weakly compact. By Gantmacher's theorem [5, p. 485] its transpose $(\Phi \underset{\mu, \varepsilon}{\otimes} \Psi)^{\prime}$ is a weakly compact operator from $\left(E_{1} \hat{\otimes} E_{2}\right)^{\prime}$ into $\left(A_{1} \bar{\otimes}_{\mu} A_{2}\right)^{\prime}$. Since the crossnorm $\pi$ is the largest of all crossnorms, the inclusion mapping $E_{1}^{\prime} \otimes E_{2}^{\prime} c^{\prime}\left(E_{1} \hat{\otimes} E_{2}\right)^{\prime}$ can be extended to an operator

$$
J: E_{1}^{\prime} \hat{\otimes} E_{2}^{\prime} \rightarrow\left(E_{1} \hat{\hat{\otimes}} E_{2}\right)^{\prime} .
$$

Let $\Theta$ be the canonical embedding from $A_{1}^{\prime} \bar{\otimes}_{\beta} A_{2}^{\prime}$ into $\left(A_{1} \bar{\otimes}_{\mu} A_{2}\right)^{\prime}$. By Lemma 2.1, $\Theta$ is an isometry. Now we have the following commutative diagram.

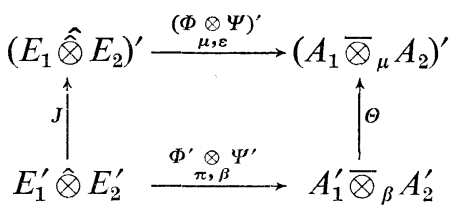

Since $\Theta$ is an isometry, $\Theta\left(A_{1}^{\prime} \bar{\otimes}_{\beta} A_{2}^{\prime}\right)$ is norm closed and convex, hence weakly closed in $\left(A_{1} \bar{\otimes}_{\mu} A_{2}\right)^{\prime}$. By the Hahn-Banach theorem the weak topology in $\left(A_{1} \bar{\otimes}_{\mu} A_{2}\right)^{\prime}$ restricted to $\Theta\left(A_{1}^{\prime} \bar{\otimes}_{\beta} A_{2}^{\prime}\right)$ corresponds via $\Theta$ to the weak topology in $A_{1}^{\prime} \bar{\oplus}_{\beta} A_{2}^{\prime}$. Hence any set in $A_{1}^{\prime} \bar{\otimes}_{\beta} A_{2}^{\prime}$ is relatively weakly compact if its image under $\Theta$ is so. Now $(\Phi \otimes \Psi)^{\prime} \circ J$ is a weakly compact operator so that the set

$$
\Theta\left(\Phi_{\pi, \beta}^{\prime} \otimes \Psi^{\prime}\left(\left(E_{1}^{\prime} \hat{\otimes} E_{2}^{\prime}\right)_{0}\right)\right)=((\Phi \underset{\mu, \varepsilon}{\otimes} \Psi) \circ J)\left(\left(E_{1}^{\prime} \hat{\otimes} E_{2}^{\prime}\right)_{0}\right)
$$

is relatively weakly compact. Consequently, also $\Phi^{\prime} \underset{\pi, \beta}{\otimes} \Psi^{\prime}$ is a weakly compact operator, and so, by Gantmacher's theorem, is its transpose

$$
\left(\Phi_{\pi, \beta}^{\prime} \Psi^{\prime}\right)^{\prime}: A_{1}^{\prime \prime} \bar{\otimes} A_{2}^{\prime \prime} \rightarrow\left(E_{1}^{\prime} \hat{\otimes} E_{2}^{\prime}\right)^{\prime}
$$

Since the dual norm of the crossnorm $\pi$ is the crossnorm $\varepsilon$, we can embed $E_{1}^{\prime \prime} \hat{\otimes} E_{2}^{\prime \prime}$ isometrically into $\left(E_{1}^{\prime} \hat{\otimes} E_{2}^{\prime}\right)^{\prime}$. Because $E_{1} \hat{\hat{\otimes}} E_{2}$ is a norm closed and convex subspace in $E_{1}^{\prime \prime} \hat{\otimes} E_{2}^{\prime \prime}$ and so also in $\left(E_{1}^{\prime} \hat{\otimes} E_{2}^{\prime}\right)^{\prime}$, it is weakly closed in $\left(E_{1}^{\prime} \hat{\otimes} E_{2}^{\prime}\right)^{\prime}$. Now $\left(\Phi^{\prime} \otimes \Psi^{\prime}\right)^{\prime}$ is $w^{*}$-w-continuous (see [5, p. 484]) and it maps the $w^{*}$-dense subset $A_{1} \otimes A_{2}$ of $A_{1}^{\prime \prime} \bar{\otimes} A_{2}^{\prime \prime}$ into the weakly closed subset $E_{1} \hat{\otimes} E_{2}$ of $\left(E_{1}^{\prime} \hat{\otimes} E_{2}^{\prime}\right)^{\prime}$. Hence we obtain

$$
\left(\Phi^{\prime} \otimes \Psi^{\prime}\right)^{\prime}\left(A_{1}^{\prime \prime} \bar{\otimes} A_{2}^{\prime \prime}\right) \subset E_{1} \hat{\hat{\otimes}} E_{2} .
$$

By the Hahn-Banach theorem the weak topology of $\left(E_{1}^{\prime} \hat{\otimes} E_{2}^{\prime}\right)^{\prime}$ induces the weak topology of $E_{1} \hat{\otimes} E_{2}$ so that $\left(\Phi^{\prime} \otimes_{\pi, \beta} \Psi^{\prime}\right)^{\prime}$ regarded as an operator into $E_{1} \hat{\otimes} E_{2}$ is weakly 
compact and $w^{*}-w$-continuous. The $w^{*}$-w-continuity of $\left(\Phi^{\prime} \otimes_{\pi, \beta} \Psi^{\prime}\right)^{\prime}$ and the fact that $A_{1} \otimes A_{2}$ is $w^{*}$-dense in $A_{1}^{\prime \prime} \otimes A_{2}^{\prime \prime}$ imply the uniqueness of $\Phi \times \Psi$, and the proof is complete.

Conversely, if we assume that $\Phi \times \Psi$ is a weakly compact operator, then $\Phi^{\prime} \bigotimes_{\pi, \beta} \Psi^{\prime}$ is weakly compact, or equivalently, $\Phi \times \Psi$ is $w^{*}$-w-continuous. Hence $\Phi \times \Psi$ maps $A_{1}^{\prime \prime} \bar{\otimes} A_{2}^{\prime \prime}$ into $E_{1} \hat{\otimes} E_{2}$. Since $\Phi \times \Psi$ extends $\Phi \underset{\mu, \varepsilon}{\otimes} \Psi$, we obtain that $\Phi \underset{\mu, \varepsilon}{\otimes} \Psi$ is also weakly compact. So we have the corollary.

Corollary 4.2. The following conditions are equivalent.

(i) The operator $\Phi \otimes \Psi$ is weakly compact.

(ii) The operator $\Phi \times \Psi$ is weakly compact.

(iii) The operator $\Phi \times \Psi$ is $w^{*}-w$-continuous.

Definition 4.3. Let $\Phi: A_{1} \rightarrow E_{1}$ and $\Psi: A_{2} \rightarrow E_{2}$ be generalized vector measures. If $\Phi \bigotimes_{\mu, \varepsilon} \Psi$ is weakly compact, the generalized vector measure $\Phi \times \Psi: A_{1}^{\prime \prime} \bar{\otimes} A_{2}^{\prime \prime} \rightarrow$ $E_{1} \hat{\otimes} E_{2}$ is called the product of $\Phi$ and $\Psi$.

In particular, we observe that if $A_{1}$ and $A_{2}$ are commutative $C^{*}$-algebras, then $\mu=\varepsilon$ on $A_{1} \otimes A_{2}$ and $\Phi \bigotimes_{\varepsilon, \varepsilon} \Psi$ is weakly compact whenever $\Phi$ and $\Psi$ are weakly compact (see e.g. [19, p. 128]) and so $\Phi \times \Psi$ is weakly compact as well.

Let $\alpha$ be any $C^{*}$-norm on $A_{1} \otimes A_{2}$. If $\Phi \bigotimes_{\mu, \varepsilon} \Psi$ is weakly compact, $\Phi \bigotimes_{\alpha, \varepsilon} \Psi$ is also weakly compact, and the generalized vector measure $\Phi \times \Psi$ is related to $(\Phi \underset{\alpha, \varepsilon}{\otimes} \Psi)^{\prime \prime}$ in the following way. Let $I:\left(A_{1} \bar{\otimes}_{\alpha} A_{2}\right)^{\prime \prime} \rightarrow A_{1}^{\prime \prime} \bar{\otimes} A_{2}^{\prime \prime}$ be the normal extension of the inclusion mapping from $A_{1} \otimes A_{2}$ into $A_{1}^{\prime \prime} \bar{\otimes} A_{2}^{\prime \prime}$ (see [18, p. 208], and [3, p. 266]). Then $(\Phi \otimes \Psi)^{\prime \prime}(x)=\Phi \otimes_{\alpha, \varepsilon} \Psi(x)=(\Phi \times \Psi) \circ I(x)$ for each $x \in A_{1} \bar{\otimes}_{\alpha} A_{2}$. So the $w^{*}$-density of $A_{1} \bar{\otimes}_{\alpha} A_{2}$ in $\left(A_{1} \bar{\otimes}_{\alpha} A_{2}\right)^{\prime \prime}$ implies that the following diagram commutes.

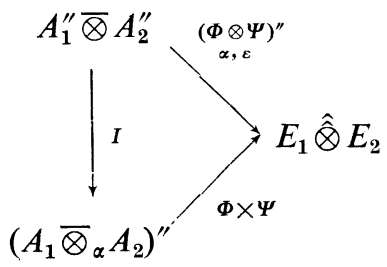

\section{Convolution}

In this section we apply the concepts developed in Sections 3 and 4 to construct and study the convolution of generalized vector measures. Throughout, $A$ is a $C^{*}$ algebra and $E_{1}$ and $E_{2}$ are any Banach spaces.

Definition 5.1. If $A$ is a $C^{*}$-algebra of type $M^{\prime}$ and $\Phi: A \rightarrow E_{1}$ and $\Psi: A \rightarrow E_{2}$ are generalized vector measures such that $\Phi \bigotimes_{\mu, \varepsilon} \Psi$ is weakly compact, then the convolu- 
tion $\Phi * \Psi$ of $\Phi$ and $\Psi$ is defined by

$$
\Phi * \Psi=(\Phi \times \Psi) \circ\left(m^{\prime} \mid A\right) .
$$

Clearly $\Phi * \Psi$ is a weakly compact operator from $A$ into $E_{1} \hat{\otimes} E_{2}$. Furthermore, it shares several properties of the convolution of ordinary regular Borel vector measures. In the following theorem we shall identify $E_{1} \hat{\hat{\otimes}} E_{2}$ with $E_{2} \hat{\hat{\otimes}} E_{1}$ and $\left(E \hat{\hat{\otimes}} E_{1}\right) \hat{\hat{\otimes}} E_{2}$ with $E \hat{\hat{\otimes}}\left(E_{1} \hat{\hat{\otimes}} E_{2}\right)$ canonically, and assume that the corresponding convolutions exist.

Theorem 5.2. Let $A$ be a $C^{*}$-algebra of type $M^{\prime}$ and $\Phi, \Psi$ and $\chi$ generalized vector measures on $A$. Then $\Phi * \Psi$ is bilinear with respect to $\Phi$ and $\Psi$. Moreover, if the multiplication of $A^{\prime}$ is commutative, then $\Phi * \Psi=\Psi * \Phi$, and if it is associative, then $\Phi *(\Psi * \chi)=(\Phi * \Psi) * \chi$.

Proof. The bilinearity follows straightly from the definition of $\Phi * \Psi$ and the bilinearity of $\Phi * \Psi$. Let the final spaces of $\Phi$ and $\Psi$ be $E_{1}$ and $E_{2}$, respectively. If the multiplication of $A^{\prime}$ is commutative, we have for each $x \in A, f \in E_{1}^{\prime}$ and $g \in E_{2}^{\prime}$

$$
\begin{aligned}
& \langle\Phi * \Psi(x), f \otimes g\rangle=\left\langle\Phi \times \Psi\left(m^{\prime}(x)\right), f \otimes g\right\rangle \\
= & \left\langle m^{\prime}(x), \Phi^{\prime}(f) \otimes \Psi^{\prime}(g)\right\rangle=\left\langle x, \Phi^{\prime}(f) \Psi^{\prime}(g)\right\rangle \\
= & \left\langle x, \Psi^{\prime}(g) \Phi^{\prime}(f)\right\rangle=\left\langle m^{\prime}(x), \Psi^{\prime}(g) \otimes \Phi^{\prime}(f)\right\rangle \\
= & \left\langle\Psi \times \Phi\left(m^{\prime}(x)\right), g \otimes f\right\rangle=\langle\Psi * \Phi(x), g * f\rangle .
\end{aligned}
$$

Since $E_{1}^{\prime} \otimes E_{2}^{\prime}$ separates the points of $E_{1} \hat{\otimes} E_{2}$, we obtain that $\Phi * \Psi=\Psi * \Phi$ with respect to the identification of $E_{1} \hat{\otimes} E_{2}$ with $E_{2} \hat{\hat{\otimes}} E_{1}$. The proof of the associativity is analogous and will be omitted.

Our motivation was to unify and generalize the concept of the convolution of ordinary regular Borel vector measures as well as that of noncommutative analogues of vector measures. The following examples show how this goal has been achieved.

Example 5.3. Let $X$ be a locally compact semigroup with a separately continuous multiplication, and let $\Phi: C_{0}(X) \rightarrow E_{1}$ and $\Psi: C_{0}(X) \rightarrow E_{2}$ be weakly compact operators corresponding to regular Borel vector measures $\bar{\mu}$ and $\bar{v}$, respectively. By Example $3.9 C_{0}(X)$ is a $C^{*}$-algebra of type $M^{\prime}$ when $C_{0}(X)^{\prime}=M(X)$ is equipped with the multiplication

$$
(\mu, v) \mapsto \mu * v \quad(\mu, v \in M(X)) .
$$

We denote the bounded linear extension of this multiplication to $M(X) \hat{\otimes} M(X)$ by $M$. The transpose of $M$ is an operator from $M(X)^{\prime}$ into $(M(X) \hat{\otimes} M(X))^{\prime}$ and for each $f \in C_{0}(X)$ and $\mu, v \in M(X)$ we have

$$
\left\langle M^{\prime} f, \mu \otimes v\right\rangle=\langle f, M(\mu \otimes v)\rangle=\langle f, \mu * v\rangle=\iint_{X} f(s t) d \mu(s) d v(t) .
$$


Further, the operator $M$ has a bounded linear extension $\tilde{M}: M(X \times X) \rightarrow M(X)$ given by

$$
\int_{X} f d \tilde{M}(\mu)=\int_{X \times X} f(s t) d \mu(s, t) \quad\left(f \in C_{0}(X)\right) .
$$

The mapping $(s, t) \mapsto f(s t)$ is integrable by [12, p. 422]. By the definition of $\tilde{M}$ its transpose $\tilde{M}^{\prime}$ restricted to $C_{0}(X)$ is the mapping $P: C_{0}(X) \rightarrow C_{0}(X \times X)^{\prime \prime}$ defined by

$$
(P(f))(s, t)=f(s t) \quad\left(f \in C_{0}(X) ; s, t \in X\right) .
$$

Let $I: M(X \times X)^{\prime} \rightarrow(M(X) \hat{\otimes} M(X))^{\prime}$ be the transpose of the inclusion mapping $J: M(X) \hat{\otimes} M(X) \rightarrow M(X \times X)$. Since $M=\tilde{M} \circ J$, we have

$$
M^{\prime}=I \circ \tilde{M}^{\prime} .
$$

Because $I$ is also the normal extension of the canonical mapping from $C_{0}(X \times X)$ into $(M(X) \hat{\otimes} M(X))^{\prime}$ (see the discussion after Definition 4.3), the following diagram commutes.

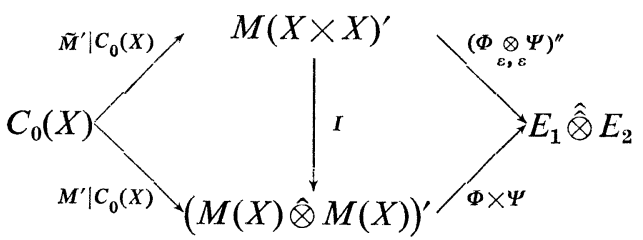

Now, the convolution of $\Phi$ and $\Psi$ as ordinary regular Borel vector measures is the composed operator $\left(\Phi \otimes_{\varepsilon, \varepsilon} \Psi\right)^{\prime \prime} \circ P$. Since $P=\tilde{M}^{\prime} \mid C_{0}(X)$, this ordinary convolution agrees with $(\Phi \times \Psi) \circ\left(M^{\prime} \mid C_{0}(X)\right)$, the convolution of $\Phi$ and $\Psi$, when they are interpreted as generalized vector measures.

Example 5.4. Let $G$ be a locally compact group and $C^{*}(G)$ its group $C^{*}$ algebra. Let $\Phi$ and $\Psi$ be noncommutative analogues of vector measures, i.e., weakly compact operators from $C^{*}(G)$ into Banach spaces $E_{1}$ and $E_{2}$, respectively. By [14, p. 25] the convolution of $\Phi$ and $\Psi$ can be defined to be the composed mapping $(\Phi \underset{v, \varepsilon}{\otimes} \Psi)^{\prime \prime} \circ p_{I}$, where $p_{I}: C^{*}(G) \rightarrow W^{*}(G \times G)$ is the restriction to $C^{*}(G)$ of the transpose $\tilde{m}^{\prime}$ of the operator $\tilde{m}: C^{*}(G \times G)^{\prime} \rightarrow C^{*}(G)^{\prime}$ given by

$$
(\tilde{m}(u))(s)=u(s, s) \quad\left(s \in G, u \in B(G \times G)=C^{*}(G \times G)^{\prime}\right) .
$$

This follows from the definition of $p_{I}$,

$$
\begin{gathered}
\int_{G} h(s, s) d f(s)=\int_{G \times G} h(s, t) d p_{I}(f)(s, t) \\
\left(h \in C_{0}(G \times G), f \in L^{1}(G)\right)[14, p p, 23-25], \text { and the equations } \\
\langle\tilde{m}(u), f\rangle=\int_{G} u(s, s) d f(s)=\int_{G \times G} u d p_{I}(f)=\left\langle u, p_{I}(f)\right\rangle,
\end{gathered}
$$

where $u \in B(G \times G)=C^{*}(G \times G)^{\prime}$ and $f \in L^{1}(G)$. Clearly $\tilde{m}$ restricted to $C^{*}(G)^{\prime} \bar{\otimes}_{\beta}$. $C^{*}(G)^{\prime}=B(G) \bar{\otimes}_{\beta} B(G)$ is the linear mapping $m$ corresponding to the multiplication 
on $B(G)$ (Example 3.10). If $I: W^{*}(G \times G) \rightarrow W^{*}(G) \bar{\otimes} W^{*}(G)$ is the transpose of the inclusion mapping $J: C^{*}(G)^{\prime} \bar{\otimes}_{\beta} C^{*}(G)^{\prime} \rightarrow C^{*}(G \times G)^{\prime}$, we obtain, as in the previous example, the following commutative diagram.

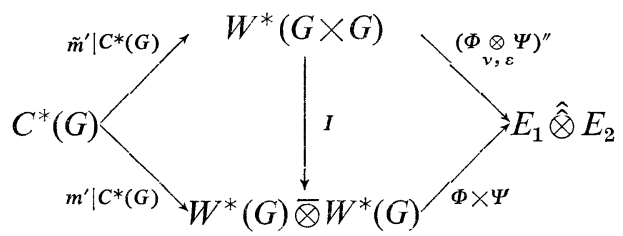

So, the convolution of $\Phi$ and $\Psi$ as generalized vector measures agrees with their convolution in the sense of [14]. Consequently, it also interacts with the Fourier transform ${ }^{\wedge}$ of [20] according to the formula $(\Phi * \Psi)^{\wedge}=\widehat{\Phi} * \hat{\Psi}$ (see [14, p. 26]).

In the previous examples the convolution can be defined via $A^{\prime \prime} \bar{\otimes} A^{\prime \prime}$ or via $\left(A \bar{\otimes}_{\alpha} A\right)^{\prime \prime}$ for some $C^{*}$-norm $\alpha$. This reflects the property that in both cases $m$ : $A^{\prime} \otimes_{\beta} A^{\prime} \rightarrow A^{\prime}$ has an extension $\tilde{m}:\left(\bar{A}_{\alpha} A\right)^{\prime} \rightarrow A^{\prime}$. However, in general it may happen that $m$ exists but $\tilde{m}$ does not. With this in mind it seems to be reasonable to construct the convolution of generalized vector measures making use of the operator $m$ : $A^{\prime} \otimes_{\beta} A^{\prime} \rightarrow A^{\prime}$.

Finally, we shall consider the convolution of generalized vector measures when the final space has a bilinear multiplication. We begin with the definition.

Definition 5.5. Let $A$ be a $C^{*}$-algebra of type $M^{\prime}$ and $B$ a Banach space of type $M_{\varepsilon}$. Denote by $m_{B}$ the operator from $B \hat{\otimes} B$ to $B$ corresponding to the multiplication of $B$. If $\Phi: A \rightarrow B$ and $\Psi: A \rightarrow B$ are generalized vector measures such that $\Phi \otimes \Psi$ is weakly compact, the operator $m_{B} \circ(\Phi * \Psi)$ is called the algebra valued convolution of $\Phi$ and $\Psi$ and denoted by $\Phi_{*}^{a} \Psi$.

So, $\Phi_{*}^{a} \Psi$ is a weakly compact operator from $A$ into $B$. The analogue of Theorem 5.2 follows immediately, and we assume again that the corresponding convolutions exist.

Theorem 5.6. Let $A$ be a $C^{*}$-algebra of type $M^{\prime}$ and $B$ a Banach space of type $M_{\varepsilon}$. Let $\Phi, \Psi$ and $\chi$ be generalized vector measures from $A$ into $B$. Then $\Phi_{*}^{a} \Psi$ is bilinear with respect to $\Phi$ and $\Psi$. Moreover, if the multiplications of $A^{\prime}$ and $B$ are commutative, then $\Phi_{*}^{a} \Psi=\Psi_{*}^{a} \Phi$, and if they are associative, then $\Phi_{*}^{a}\left(\Psi_{*}^{a} \chi\right)=\left(\Phi_{*}^{a} \Psi\right) \stackrel{a}{*} \chi$.

The algebra valued convolution induces a multiplication on several operator spaces, for the case when $A=C^{*}(G)$ see [14, Chapter 5]. Here we shall consider as an example the space $\mathscr{K}(A, B)$ of compact operators from $A$ into $B$. (An operator $\Phi: A \rightarrow B$ is called compact if $\Phi\left(A_{0}\right)$ is relatively compact in $B$.)

Lemma 5.7. If $\Phi: A_{1} \rightarrow E_{1}$ and $\Psi: A_{2} \rightarrow E_{2}$ are compact operators, then $\Phi * \Psi$ is compact. 
Proof. By [10, p. 401] $\Phi \underset{\varepsilon, \varepsilon}{\otimes} \Psi$ is compact if $\Phi$ and $\Psi$ are compact. Hence $\Phi \underset{\mu, \varepsilon}{\otimes} \Psi$ is compact so that by Schauder's theorem [5, p. 485] $(\Phi \underset{\mu, \varepsilon}{\otimes} \Psi)^{\prime}$ is compact. Using the commutative diagram in the proof of Theorem 4.1 we now deduce that $\Phi^{\prime} \otimes_{\pi, \beta} \Psi^{\prime}$ is a compact operator and so is also its transpose $\Phi \times \Psi$. The assertion follows now straightly.

It is easy to see that $\|\Phi \times \Psi\| \leqq\|\Phi\|\|\Psi\|$ so that $\|\Phi * \Psi\| \leqq\|\Phi\|\|\Psi\|\|m\|$. If $A^{\prime}$ and $B$ are Banach algebras of type $M_{\beta}$ and $M_{\varepsilon}$ with $\|m\| \leqq 1$ and $\left\|m_{B}\right\| \leqq 1$, respectively, then $\mathscr{K}(A, B)$ equipped with the algebra valued convolution becomes a Banach algebra. It is easy to see that in all the examples given in Section 3 we have $\|m\| \leqq 1$.

If $A^{\prime}$ or $B$ has the (Grothendieck) approximation property, then $\mathscr{K}(A, B)$ is isometrically isomorphic to $A^{\prime} \dot{\otimes} B[16$, p. 113], which therefore becomes a Banach algebra. Moreover, if $A^{\prime}$ and $B$ are commutative, we can apply the theorem of Gelbaum and Tomiyama [13, p. 179], which states that if $A^{\prime} \hat{\otimes} B$ is a Banach algebra, then its spectrum is homeomorphic to $\sigma\left(A^{\prime}\right) \times \sigma(B)$.

Theorem 5.8. Let $A$ be a $C^{*}$-algebra of type $M^{\prime}$ such that $A^{\prime}$ is a Banach algebra. Let $B$ be a Banach algebra of type $M_{\varepsilon}$. If the operator norms of $m$ and $m_{B}$ are less than or equal to one, then $\mathscr{K}(A, B)$ is a Banach algebra. Moreover, if $A^{\prime}$ and $B$ are commutative and one of them has the approximation property, then $\sigma(\mathscr{K}(A, B))$ is homeomorphic to $\sigma\left(A^{\prime}\right) \times \sigma(B)$.

This theorem can be regarded as a generalization of a result of P.S. Chow [1, p. 118]. He has proved that $\sigma\left(\mathscr{K}\left(C_{0}(G), C(K)\right)\right)$ is homeomorphic to $\sigma\left(C_{0}(G)^{\prime}\right) \times$ $\sigma(C(K))$, where $G$ is a locally compact commutative group and $K$ a compact space.

Acknowledgement. This research has been supported by the Emil Aaltonen foundation.

\section{References}

[1] CHow, P. S.: On some Banach algebras of measures. - Ph. D. Thesis, Aberdeen University, 1969.

[2] Civin, P., and B. Yood: The second conjugate space of a Banach algebra as an algebra. Pacific J. Math. 11, 1961, 847-870.

[3] Dixmier, J.: C*-algebras. - North-Holland Mathematical Library Vol. 15, North-Holland Publishing Company, Amsterdam-New York-Oxford, 1977.

[4] DuchoŇ, M.: Biprojective tensor products and convolutions of vector-valued measures on a compact group. - Studia Math. 38, 1970, 187--192.

[5] Dunford, N., and J. T. Schwartz: Linear operators, Part I: General theory. - Pure and Applied Mathematics 7, Interscience Publishers, Inc., New York-London, 1958.

[6] Ernest, J.: Hopf-von Neumann algebras. - Proceedings of the Conference on Functional Analysis, Irvine, California 1966, edited by B. R. Gelbaum, Thompson Book Company, Washington, D. C., Academic Press, London, 1967, 195-215.

[7] Eymard, P.: L'algèbre de Fourier d'un groupe localement compact. - Bull. Soc. Math. France 92, 1964, 181-236. 
[8] Glicksberg, I.: Weak compactness and separate continuity. - Pacific J. Math. 11, 1961, $205-214$.

[9] Guichardet, A.: Tensor products of $\mathrm{C}^{*}$-algebras. - Aarhus Universitet, Matematisk Institut, Lecture Notes Series 12, 1967, 1-55.

[10] Holub, J. R.: Compactness in topological tensor products and operator spaces. - Proc. Amer. Math. Soc. 36, 1972, 398-406.

[11] De MAgalhães IóRIo, V.: Hopf-C*-algebras and locally compact groups. - Pacific J. Math. $87,1980,75-96$.

[12] Johnson, B. E.: Separate continuity and measurability. - Proc. Amer. Math. Soc. 20, 1969, $420-422$.

[13] Mallios, A.: On the spectrum of a topological tensor product of locally convex algebras. Math. Ann. 154, 1964, 171-180.

[14] Martikainen, O.: Convolutions of noncommutative analogues of vector mesures with applications to operator ideals. - Ann. Acad. Sci. Fenn. Ser. A I Math. Dissertationes $18,1978,1-65$.

[15] Rickart, C. E.: General theory of Banach algebras. - D. Van Nostrand Company, Inc., Princeton, New Jersey-Toronto-London-New York, 1960.

[16] Schaefer, H. H.: Topological vector spaces. - The Macmillan Company, New York--London, 1966.

[17] Semadeni, Z.: Banach spaces of continuous functions. - PWN-Polish Scientific Publishers, Warszawa, 1971.

[18] TAKesaki, M.: Theory of operator algebras I. - Springer-Verlag, New York-HeidelbergBerlin, 1979.

[19] White, A. J.: Convolution of vector measures. - Proc. Roy. Soc. Edinburgh Sect. A 73, 1975, 117-135.

[20] YLINEN, K.: Fourier transforms of noncommutative analogues of vector measures and bimeasures with applications to stochastic processes. - Ann. Acad. Sci. Fenn. Ser. A I Math. 1, 1975, 355-385.

University of Oxford

Mathematical Institute

Oxford 0X1 3LB

England
Helsinki University of Technology Institute of Mathematics

SF-02150 Espoo 15

Finland

Received 8 June 1982 